\title{
Diagnosis and Management of Intraductal Papillary Mucinous Neoplasm of the Pancreas Where Tropical Calcific Pancreatitis Is Prevalent
}

\author{
Prakash Kurumboor*, Kamalesh NP, Pramil K, Deepak George and RohanShetty, Shaji P \\ Department of GI Surgery, Digestive Diseases Center, PVS Memorial Hospital, Kaloor, Kochi, Kerala, India
}

Received: November 13, 2017; Accepted: January 5, 2017; Published: April 25, 2017

*Corresponding author: Prakash Kurumboor, Chief GI Surgeon, Dept. of GI Surgery, PVS Memorial Hospital, Kaloor, Kochi, Kerala, India; Tel: 0091484 2345451 (or) 09447302588; E mail: kdrprakash@gmail.com

\begin{abstract}
Intraductal Papillary Mucinous Neoplasm of Pancreas (IPMN) is being more frequently diagnosed and treated worldwide as clinicopathological features of this entity is well described. The entity IPMN was included in the WHO classification system in 1996 and is classified as branch duct IPMN and main duct disease essentially based on radiological features. This disease is considered relatively rare in India, except for a couple of published case reports. Tropical pancreatitis is a form of idiopathic chronic pancreatitis seen in tropical Asia and Africa. This entity is characterised by obstruction of the pancreatic duct by a stricture or stone with upstream dilatation of the duct, the features which have similarities with IPMN making accurate preoperative diagnosis of IPMN difficult. This article describes the diagnosis, surgical treatment and pathological details of these patients and the difficulties in diagnosing IPMN in areas where tropical pancreatitis is prevalent.
\end{abstract}

\section{Introduction}

WIntraductal Papillary Mucinous Neoplasm of Pancreas (IPMN) was first described by Ohashi et al in 1982 [1]. This disease is being increasingly recognized and managed worldwide following the WHO's description of the disease, its nomenclature and its pathological characteristics [2,3]. Essentially, the disease is classified into Branch duct type and Main duct Type. Any dilatation of the main pancreatic duct (MPD) $>5 \mathrm{~mm}$ without other causes of obstruction has been identified significant thereby increasing the sensitivity for radiologic diagnosis without losing specificity [4]. It is well identified that there is increased risk for malignancy in IPMN and may result in invasive adenocarcinoma and hence considered as a premalignant condition. Even with development if invasive carcinoma in IPMN, the prognosis is better than pancreatic ductal adenocarcinoma and hence surgical management is adopted whenever feasible.5 Hence, proper diagnosis of this disease is important for proper planning of the treatment especially surgery. In India, the disease prevalence data is not yet available except for few case reports of the disease $[6,7]$. In tropical Asia and Africa, tropical chronic pancreatitis (TCP), a form of idiopathic chronic pancreatitis is prevalent8. This entity is characterised by obstruction of the pancreatic duct by a stricture or stone with upstream dilatation of the duct. These clinical features which have similarities with IPMN particularly when the stone load is sparse in tropical pancreatitis. This makes accurate preoperative diagnosis of IPMN difficult. Rarely both these disease forms can coexist as well [6]. This retrospective study is a descriptive analysis of the diagnosis, surgical treatment and pathological details of these patients and the difficulties in diagnosing IPMN in areas where tropical pancreatitis is prevalent.

\section{Methods}

From May 2009 to October 2014, twenty-four patients with IPMN were operated in our unit. There were 16 males and 8 females with median age of 58 years. Abdominal pain, weight loss and or jaundice were the clinical presentation. Patients were investigated using ultrasonography, contrast enhanced CT scan and endosonography (EUS). EUS guided FNAC were obtained from suspicious lesions. EUS guided FNA was used in cases with mass lesions/mural nodules. CT scan findings were suggestive of IMPN in $6(25 \%)$ of patients and that in endosonography was 13 (54\%) (Table 1).

\begin{tabular}{|c|c|}
\hline $\begin{array}{l}\text { Table 1: Demographic features, preoperative and postoperative } \\
\text { findings }\end{array}$ & Number (\%) \\
\hline Parameter (N=24) & $16: 08$ \\
\hline Male: female & 58 years \\
\hline Age (median) & $19(79 \%)$ \\
\hline Clinical presentation & $12(50 \%)$ \\
\hline Abdominal pain & $6(25 \%)$ \\
\hline Weight loss & $14(58 \%)$ \\
\hline Jaundice & \\
\hline Back pain & Investigations \\
\hline & CT Scan Findings \\
\hline IPMN & $6(25 \%)$ \\
\hline Cystic neoplasm/dilated PD & $7(29)$ \\
\hline
\end{tabular}




\begin{tabular}{|c|c|}
\hline CCP head mass & $6(25 \%)$ \\
\hline Head mass with dilated PD & $5(21 \%)$ \\
\hline \multicolumn{2}{|c|}{ Endosonography Findings } \\
\hline IPMN & $13(54 \%)$ \\
\hline CCP head mass & $5(21 \%)$ \\
\hline Head mass with dilated PD & $3(12 \%)$ \\
\hline Cystic neoplasm & $3(12 \%)$ \\
\hline Preoperative diagnosis (Clinical+ imaging findings) \\
\hline IPMN/suspicious of IMPN & $15(62.5 \%)$ \\
\hline CCP head mass & $5(20.8 \%)$ \\
\hline Head mass with dilated PD & $4(16.7 \%)$ \\
\hline \multicolumn{2}{|c|}{} \\
\hline Preoperative diagnosis of \\
malignancy
\end{tabular}

Mural nodules were identified in 7 (29\%) in EUS and malignant degeneration was diagnosed by EUS guided FNAC in 9(37.5\%) patients. Taking into clinical features and radiological investigations, a preoperative provisional diagnosis of IPMN was made in 15 (62.5\%). In the remaining 5 patients preoperative diagnosis was TCP with head mass in 5 (20.8\%) and head mass suspicious of malignancy in $4(16.7 \%)$.

At surgery 18 patients underwent pancreatoduodenectomy, distal pancreatectomy in 2 patients and 4 patients underwent total pancreatectomy. All patients who underwent pancreatoduodenectomy underwent frozen section to assess the adequacy of the resection. Four patients required portal vein resection and 2 patient's required additional resection in the form of gastrectomy and transverse colectomy. There were 16 (66.6\%) patients with branch duct IPMN and 8 $(33.3 \%)$ patients with main duct IPMN. Malignant degeneration was noted in16 (66.6\%) patients. Noticeably, malignancy with IPMN was common in those patients with mass lesion and dilated duct. All patients had negative resection margins in the final histopathology, except one patient with main duct disease and mass head of pancreas that underwent pancreatoduodenectomy had a positive resection margin status. Postoperative morbidity was observed in one patient due to delayed gastric emptying. There was no mortality in the cohort and the mean hospital stay was 11.4 days.

\section{Discussion}

Intraductal mucinous neoplasm of pancreas is being diagnosed more frequently as the clinicopathological and radiological features of this condition is better described in recent literature $[3,4,9]$. Further, MPD dilation of $5-9 \mathrm{~mm}$ is considered a "worrisome feature", while an MPD diameter of "10 $\mathrm{mm}$ is one of the "high-risk stigmata" warranting further evaluation by endosonography [4]. In India, the incidence of this condition IPMN is not clearly known, apart from few case reports. Since TCP is prevalent any patient with obstruction of the pancreatic duct by a stricture or stone with upstream dilatation of the duct is considered as chronic pancreatitis even when the stone load is sparse [8]. In the light of this series, patients presenting symptoms and with dilated pancreatic duct, cystic dilatation or mass lesion with pancreatic duct one should keep a differential diagnosis of IPMN. In our experience even after cross sectional imaging and EUS, it is difficult to attain a single final diagnosis as commonest differential diagnosis being tropical pancreatitis and one should keep IPMN as a possibility in such a clinical situation. In the current series, preoperative suspicion of IPMN was made in $62.5 \%$ of patients and in the rest preoperative diagnosis were tropical calcific pancreatitis or cystic neoplasm of pancreas. It has been noted that in $12-60 \%$ of cases preoperative diagnosis of chronic pancreatitis is considered and in about $2 \%$ of cases chronic pancreatitis is associated with IPMN $[9,10]$. In India, especially in areas where tropical pancreatitis is prevalent and this entity may co-exist with IPMN, this poses a challenge for the clinicians to make a diagnosis of IPMN as this entity is being recognised recently in our patients [6]. Identification of this disease entity is particularly important as the prognosis of IPMN even with invasive adenocarcinoma is considered better than pancreatic ductal adenocarcinomas. Conversely, identification of IPMN would alert the clinician to assess these patients for potential malignant degeneration, which would have an impact on the long term outcome of these patients.

Predominant type of disease in this series was branch duct type in this series and malignant degeneration was noted in $66 \%$ of patients. Presence of dilated pancreatic duct with mural nodule is often correlated with malignant degeneration in these patients. Hence, imaging and targeted biopsy using EUS FNA in suspicious cases is very useful for planning treatment. EUS and guided FNACs help to identify those lesions with 'worrisome features and high risk stigmata. In this series eighteen patients underwent pancreatoduodenectomy and two patients had distal pancreatectomy and total pancreatectomy was required in 4 patients. Two patients who had total pancreatectomy had features indistinct from tropical pancreatitis and malignant degeneration of head region as well as body of pancreas (Figure 1).Thorough preoperative assessment, keeping a high of suspicion during surgery and appropriate use of frozen section to assess resection margins are important step to have optimum treatment of IPMN patients. 


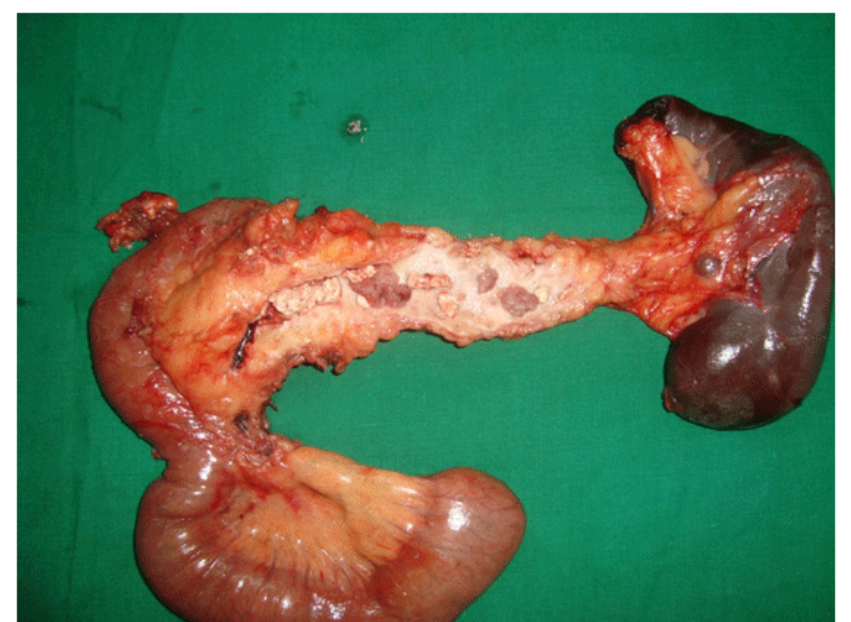

Figure 1: Tropical pancreatitis and malignant degeneration of head region as well as body of pancreas

To conclude, IMPN is being increasingly identified in India. As tropical pancreatitis is prevalent in India, every effort should be made to differentiate IPMN patients and to identify those with malignant degeneration. EUS and guided biopsy helps to diagnose these lesions correctly and to identify those with malignant degeneration.

\section{References}

1. Ohashi K, Murakami Y, Takekoski T. Four cases of 'mucin producing' cancer of the pancreas on specific findings of the papilla of Vater. Prog Dig Endosc. 1982;20:348-351.

2. Klöppel G, Solcia E, Longnecker DS, Capella C, Sobin L. Histologic typing of tumors of the exocrine pancreas. In: World Health Organization ed. International Classification of Tumors. 2nd ed. Berlin: Berlin: Springer, 1996:11-20.
3. Longnecker DS, Adler G, Hruban RH, Klöppel G. Intraductal papillary mucinous neoplasms of the pancreas. In: World Health Organization Classification of Tumors. Pathology and Genetics of Tumors of the Digestive System. Lyon, IARC Press. 2000:237-241.

4. Tanaka M, Chari S, Adsay V, Fernández-del Castillo C, Falconi M, et al. International Association of Pancreatology. International consensus guidelines for management of intraductal papillary mucinous neoplasms and mucinous cystic neoplasms of the pancreas. Pancreatology. 2006;6(1-2):17-32.

5. Poultsides GA, Reddy S, Cameron JL, Hruban RH, Pawlik TM, Ahuja N, et al. Histopathologic basis for the favorable survival after resection of intraductal papillary mucinous neoplasm associated invasive adenocarcinoma of the pancreas. Ann Surg. 2010;251(3):470-476. doi: 10.1097/SLA.0b013e3181cf8a19

6. Ramesh H, Jacob M, Mahadevan P. Intraductal papillary mucinous neoplasm in tropical chronic pancreatitis Indian J Gastroenterol. 2007;26(3):139-140.

7. Simon E, Joseph AJ, Choudhrie L, Eapen A, Vyas F, Sitaram V, et al. Intraductal papillary mucinous neoplasm of the pancreas. Indian J Gastroenterol. 2010;29:46.

8. Balakrishnan V, Unnikrishnan AG, Thomas V, Choudhuri G, Veeraraju P, Singh SP, et al. Chronic pancreatitis. A prospective nationwide study of 1086 subjects from India. JOP. 2008;9(5):593-600.

9. Serikawa M, Sasaki T, Fujimoto Y, Kuwahara K, Chayama K. Management of intraductal papillary-mucinous neoplasm of the pancreas: treatment strategy based on morphologic classification. J ClinGastroenterol. 2006;40(9):856-862.

10. Loftus EV Jr, Olivares-Pakzad BA, Batts KP, Adkins MC, Stephens DH, Sarr MG, et al. Intraductalpapillary mucinous tumors of the pancreas: clinicopathologic features, outcome, and nomenclature. Members of the Pancreas Clinic, and Pancreatic Surgeons of Mayo Clinic. Gastroenterology. 1996;110(6):1909-1918.

11. Talamini G, Zamboni G, Salvia R, Capelli P, Sartori N, Casetti L, et al. Intraductal papillary mucinousneoplasms and chronic pancreatitis. Pancreatology. 2006;6(6):626-634. 